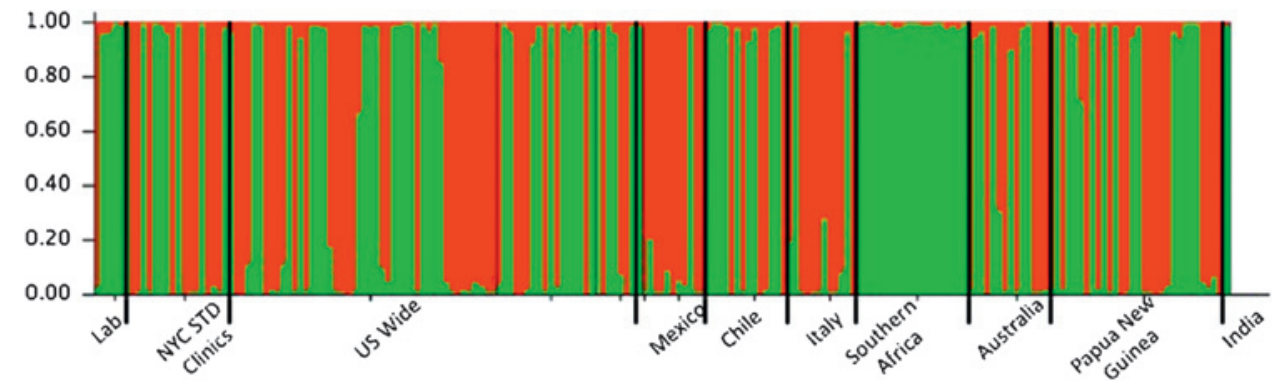

Abstract P4-S3.05 Figure 1 Two-phylotype population structure of global Trichomonas vaginalis isolates.

$20^{\circ} \mathrm{C}$, male samples were $100 \%$ reactive after 7 days storage, while female samples were $100 \%$ reactive after 6 days of storage. For samples stored at $30^{\circ} \mathrm{C}, 100 \%$ reactivity was obtained at 2 days storage for male samples and 4 days storage for female samples.

Conclusions This study shows urine samples from women infected with TV have a wide range of cell titres, with an average of $\sim 2000$ TV cells $/ \mathrm{ml}$. TV can be detected for 14 days when stored refrigerated or for about 1 week at $20^{\circ} \mathrm{C}$. The use of sensitive, automated molecular tests such as the ATV assay for testing urine samples should facilitate screening for TV.

\section{P4-S3.06 STUDY ON EARLY INFECTION OF THE LOW GENITAL TRACT OF FEMALE MICE BY DIFFERENT SEROTYPES UREAPLASMA UREALYTICUM}

doi:10.1136/sextrans-2011-050108.521

B Yang. Guangdong Provincial Center for Skin Disease and STD Control, Guangzhou, China

Objective To observe the early inflammatory response by animal model with low genital tract $U u$ infection for investigating the relationship between the serovars and their pathogenicity.

Methods Fifty 8-10-week-old, female BALB/c mice were randomly divided into five groups, namely, control group, serovar 1 group, serovar 3 group, serovar 4 group, and serovar 8 group, and then treated respectively with vaginal inoculation of liquid medium and Uu inoculae of serovars 1, 3, 4 and 8 after subcutaneous injection of oestradiol benzoate. The vulval symptoms and vaginal and cervical swabs were observed weekly after Uu inoculation. Four weeks later, all mice were sacrificed and their cervixes and vaginae were removed for histopathology.

Results There was a significant difference in the inflammation severity of cervical mucosa $(p<0.001-0.05)$ except vaginal mucosa among all groups. The inflammation severity of cervical mucosa in serovar 4 and 8 infection was superior to that in serovar 1 infection $(p<0.01-0.05)$, but there was no statistical difference among other groups ( $p>0.05)$.

Conclusions The results suggest that Uu could colonise and infect the cervical columnar epithelium other than vaginal squamous epithelium. Uu-induced inflammation response is different among its serovars. Serovars 4 and 8 are more virulent compared with biovar 1 such as serovar 1 .

\section{P4-S3.07 POPULATION GENOMICS OF TRICHOMONAS VAGINALIS REVEALS A GLOBALLY DISTRIBUTED TWO-PHYLOTYPE POPULATION STRUCTURE}

doi:10.1136/sextrans-2011-050108.522

${ }^{1} \mathrm{M}$ Conrad, ${ }^{1} \mathrm{~A}$ Gorman, ${ }^{2} \mathrm{~J}$ Schilinger, ${ }^{1} \mathrm{~S}$ Sullivan, ${ }^{3} \mathrm{~J}$ Upcroft, ${ }^{4} \mathrm{~J}$ Gonzalez, ${ }^{5} \mathrm{P}$ L Fiori, ${ }^{6} \mathrm{R}$ Arroyo, ${ }^{7} \mathrm{~J}$ Ravel, ${ }^{8} \mathrm{~W}$ E Secor, ${ }^{1} \mathrm{~J}$ Carlton. ${ }^{1}$ New York University School of Medicine, New York, USA; ${ }^{2}$ New York City Department of Health and Mental Hygeine, USA; ${ }^{3}$ Queensland Institute of Medical Research, Australia; ${ }^{4}$ University of Antofogasta, Chile; ${ }^{5}$ University of Sassari, Italy; ${ }^{6}$ Centro de Investigacion y de Estudios Avanzados del IPN,
Mexico; ${ }^{7}$ University of Maryland, School of Medicine, USA; ${ }^{8}$ Centers for Disease Control and Prevention, USA

Objective Trichomonas vaginalis, the causative agent of human trichomoniasis, is the most prevalent non-viral sexually transmitted infection and has been associated with increased risk of HIV transmission, making detection and treatment a global health priority. In this study, we evaluate the population genomics of globally distributed clinical isolates to characterise genetic diversity and identify population structure.

Methods We use a panel of 21 microsatellite and three single copy gene markers to evaluate the population genomics of 18 clinical isolates collected from female patients attending New York City STD clinics in 2008, as well as 177 extant isolates collected from the USA, Mexico, Chile, Italy, Southern Africa, Papua New Guinea, and Australia. We use a panel of population genetic tools including Arlequin 3.11 and FSTAT to calculate expected heterozygosity (HE) and population differentiation (FST) statistics. To infer population structure, we use STRUCTURE 2.2, Network 4.516, and SeaView 4.2.4. We test for significance in clinical and demographic variables $\left(\chi^{2}\right.$ and t-tests) using JMP Genomics 5.0.

Results We detect significant genetic diversity within the species $(\mathrm{HE}=0.66)$ that is observed across global regions (range 0.52-0.67), and find that a two-phylotype population structure is maintained globally with few instances in population differentiation defined by geographical origin. This two-phylotype structure is further supported by minimum spanning networks, hierarchical clustering and phylogenies inferred from three single copy genes. These two phylotypes appear at nearly equal frequencies globally. Defining characteristics of the phylotypes include significantly different prevalence of $T$ vaginalis virus infection ( $3 \%$ in phylotype 1 and $73 \%$ of phylotype 2 ( $p<0.0001$, $\left.\chi^{2}\right)$ ) see Abstract P4-S3.07 figure 1 and a significantly higher minimum inhibitory concentration of metronidazole in phylotype $1(p=0.0024$, $\chi^{2}$ Wilcoxon Rank Sums)

Conclusions We detect clear population structure and high levels of genetic diversity from global $T$ vaginalis isolates. This preliminary data

\section{TVV Infection Status by Phylotype}

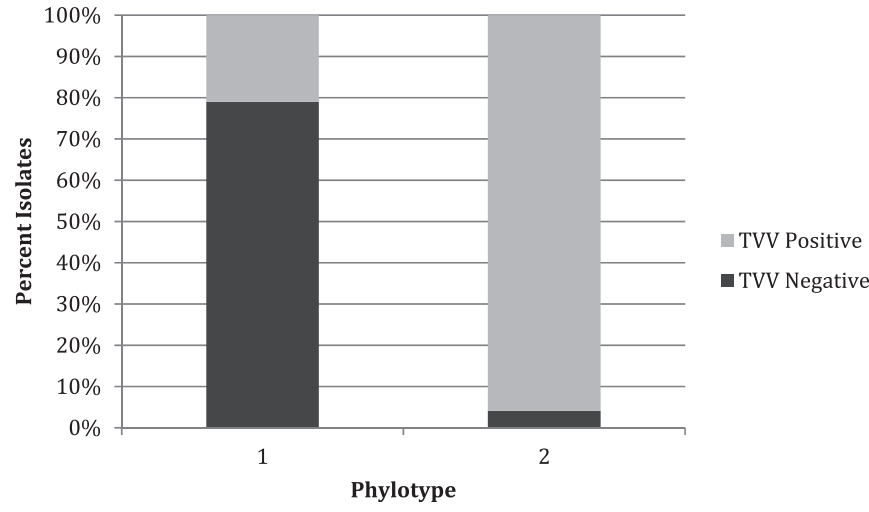

Abstract P4-S3.07 Figure 1 TVV infection status by phylotype. 